\title{
An Enhanced MAC Architecture for Multi-hop Wireless Networks
}

\author{
R. Bernasconi, I. Defilippis, S. Giordano, and A. Puiatti \\ University of Applied Sciences, (SUPSI), \\ Computer Science and Electrical Engineering Department, \\ 6928 Manno, Switzerland \\ \{bernascr, ivan.defilippis, silvia.giordano, puiatti\} \\ adie.supsi.ch \\ http://www.die.supsi.ch
}

\begin{abstract}
The IEEE 802.11 MAC family of standards has been designed for communication in traditional wireless LANs. The enhancement of the IEEE 802.11 family of standards is mainly seen in terms of channel speeds [1][2]. This fact, combined with advances in portable/handable computing and wireless technologies are expected to further increase possibilities and significant interest in the design and development of instantly deployable, wireless networks. Its usability in multi-hop wireless networks has been discussed in several papers. Starting from several known problems and issues, and analyzing the cause of these problems, we design the architecture of an enhanced IEEE 802.11 MAC card for multi-hop wireless networks ${ }^{1}$.
\end{abstract}

\section{Introduction}

Instantly deployable, multi-hop wireless networks can be both fully self-organized (ad-hoc networks) and connected to the wired backbone via multiple wireless hops (multi-hop wireless LANs). Potential examples of this include rescue, citizen and guerrilla networks, where users self-organize to communicate or to access the Internet; multi-hop Hot Spots, wireless networks in public areas where traditional WLAN technology is augmented with ad-hoc wireless communications. However, several recent works [3], [4], [5], expressed serious doubt about the workability of IEEE 802.11 in real multi-hop scenarios. In this paper, we propose the architecture for an enhanced IEEE 802.11 MAC card to seamlessly work in a multi-hop wireless network, but, at the same time, it is still fully compatible with traditional IEEE 802.11 implementations. This architecture is designed to address: 1) a backoff scheme more adequate to multi-hop mode; 2) channel access and switching; 3) routing performed at MAC layer; 4) cross-layering.

1 This work was partially funded by the Information Society Technologies programme of the European Commission, Future and Emerging Technologies under the IST-2001-38113 MOBILEMAN project. 


\section{Problems Definition}

The 802.11 MAC defines two different shared access methods to the wireless medium, the Distributed Coordination Function (DCF), described as support for ad hoc architectures - where each node acts a peer to all nodes within its transmission range, and the Point Coordination Function (PCF). The DCF method, which involves a purely distributed mechanism for contention resolution, is indeed employed in ad hoc and multi-hop wireless networks, even if does not present any real forwarding capability. DCF is mainly a carrier sensing with collision avoidance mechanism: station $\mathrm{N} 1$, in order to send to station N2, uses a 4-way handshake mechanism (request-to-send - RTS, clear-to-send - CTS, Data, Ack) to both avoid collisions and verify reliable packet forwarding. If the medium is free for a specified time (Distributed Inter Frame Space - DIFS), then the station is allowed to transmit. The exponential backoff mechanism is further used after collision, to solve contention situations. If N1 senses the medium busy then it defers for a random backoff time in the range ( 0 , Congestion Window). Upon failure of the RTS packet, the congestion window is doubled. The backoff timer is decreased whenever the channel is free; the node makes a fresh attempt at sending an RTS packet upon the expiration of the timer. These mechanisms, designed for WLAN environments, do not work well with multi-hop mode.

Backoff: In the 802.11 MAC, the channel access scheme is regulated by the exponential backoff: nodes failing to obtain the channel have to backoff a random time before trying again. It has been shown in [3], [4] that, depending on the network configuration, the standard IEEE 802.11 protocol can be not fair and operate very far from the theoretical limit of the wireless network. Both unfairness and low channel utilization are reflected higher, especially at transport layer, if TCP is used. This was shown in simulations [3], [4], and resulted even worst when tested in real experiments [5]. On the opposite, the capacity of an IEEE 802.11 protocol with a backoff window tuned on the optimal value is fairer and close to the theoretical limit.

Channels: Traditionally, IEEE 802.11 implementations allow the use of a small set of channels (usually channels 10 and 11). There are also serious difficulties to switch from one channel to another at a single node (it is necessary to tear down the network interface, modify the operating channel in the configuration file and then restart it). In order to fully exploit the capacity of multi-hop mode (see, for example [6]) and reduce contention under network congestion status, it is important to work with multiple operating channels and easily switch from one to another.

Routing: The main motivation for integrating MAC and forwarding functionality is highlighted in [7] and arises out of the fact that wireless nodes have conventionally just one interface, which is involved twice during the forwarding process. That is: a packet received at the wireless interface, it must be passed up to the routing layer (in order to discover the next hop), and further down to the same wireless interface for transferring it to the next hop. This adds undesirable delay and overhead at both MAC and routing layer.

Cross-layering: In several recent works [8], [9], [10]; the authors point out the need of a cross-layering design. More than routing, research has shown that several mechanisms can profit by the knowledge of some parameters that are typically confined at the MAC: transport, power management, cooperation, etc... 


\section{Features of the Enhanced Architecture}

The main innovative characteristics of our implementation, as illustrated in Figure 1, are: P-persistent IEEE 802.11, Multi-channels communication mode, MAC packet forwarding, Exportable parameters module.

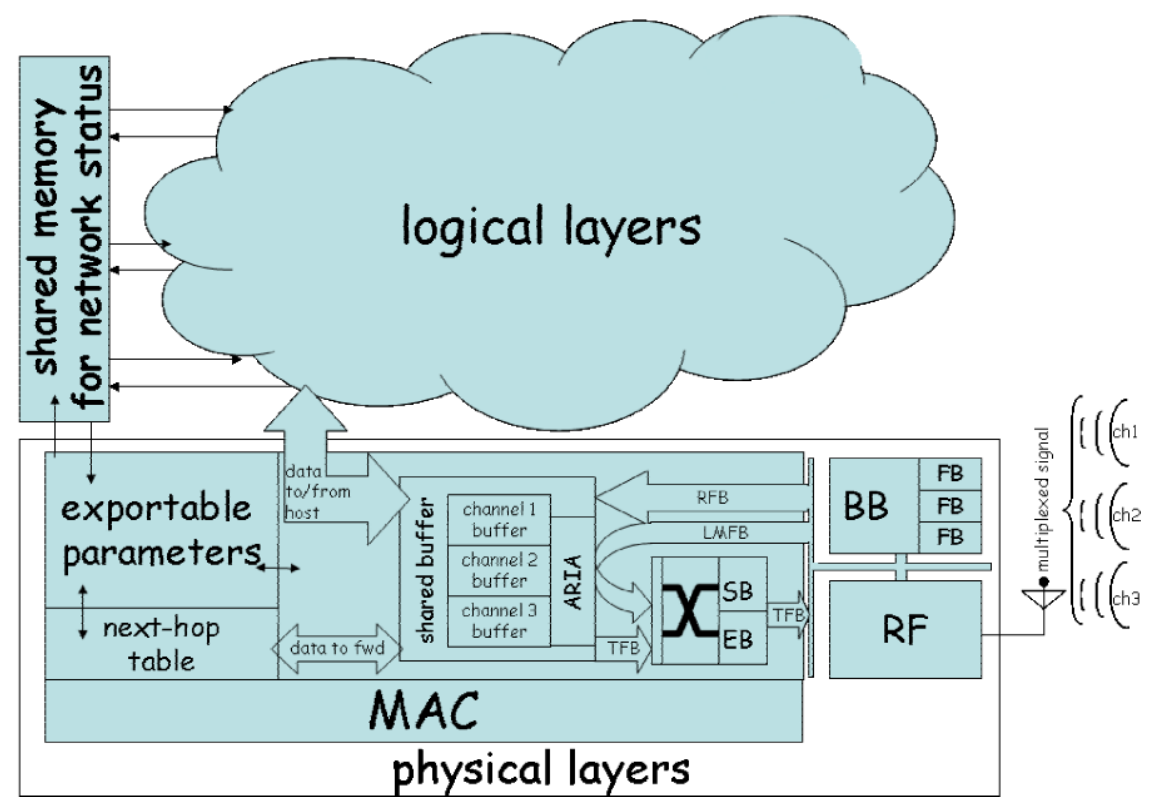

Fig. 1. Enhanced MAC card architecture. Multiple transmissions (up to 3 non-interfering channels) can be received as multiplexed signal at the antenna. The BaseBand (BB) receives it from the Radio Frequency (RF), and demultiplexes it towards up to 3 Frame Buffers (FB). The content of each FB is checked as usual (with CRC) and sent it to MAC on the Received Frames Bus (RFB) or on the Local Managed Frames Bus (LMFB). For a frame received on LMFB, the Additional Routing Information Analyzer (ARIA) checks if it contains some information for further forwarding. If so, it contacts the Next Hop Table to discover the next hop. The MAC has to manage the contention by assigning different priorities and handling the communication on the different channels (bufferized in separate memory spaces). The transmission is sent to the BB over the Transmitted Frames Bus (TFB) following the active backoff algorithm: Standard Backoff (SB), which implements the standard IEEE 802.11, or Enhanced Backoff (EB)

P-persistent IEEE 802.11: Several works have shown that, with an opportune tuning of the backoff algorithm, the protocol capacity increases [12] [13]. Our card will have the possibility to switch from standard IEEE 802.11 backoff to a backoff more adapt for the multi-hop mode. Our design is based on algorithms for approaching the performance of the p-persistent protocol [4]. The p-persistent IEEE 802.11 protocol differs from the standard protocol only in the selection of the backoff interval. Instead of the binary exponential backoff used in the standard, the backoff interval of the ppersistent IEEE 802.11 protocol is sampled from a geometric distribution with 
parameter p. In [4] it was shown that the p-persistent IEEE 802.11 protocol closely approximates the standard protocol (at least from the protocol capacity standpoint) if the average backoff interval is the same.

Multi-channels communication mode: our card can use all the available standard channels and switch from one channel to another in order to increase the node performances and the bandwidth utilization. In the Direct Sequence (DS) transmission mode the IEEE 802.11 standard divides the bandwidth in 14 channels spaced by 5 $\mathrm{MHz}$ with a bandwidth of about $22 \mathrm{MHz}$ for each channel. It means that in a multiple cell network topology, overlapping and/or adjacent cells using different channels can operate simultaneously without interference if the distance between the center frequencies is at least $30 \mathrm{MHz}$. This already limits the performance of several nodes in the same area. Suppose we have 3 groups of nodes wishing to communicate in the same area without interfering: if group1 is using channel 1, group2 can use any channel equal to or larger than channel 7 (i.e. channel $7,8, \ldots$ ), while group3 can communicate without interferences only if group2 uses channel 7 or channel 8, using channel 13 or channel 14 respectively. This can be seen as a limitation to the flexibility of the system as, for example, channel 7 and channel 8 could be not usable for group2, while a channel communicating at a frequency between 2.448 and 2.451 $\mathrm{GHz}$ could be. The new design includes:

- Maximize the flexibility of use of the bandwidth by setting each carrier frequency with a step of $1 \mathrm{MHz}$;

- Multiplexed channel sensing to receive transmissions on multiple channels; multiple buffers baseband, to manage multiple frames; multiple backoff computation (that allows to choose the channel with smaller backoff in homogeneous enhanced MAC-based scenarios);

- Priority mechanism for traffic on different channels. This includes the introduction of new broadcast messages for alerting the other possible channels in case of scenarios that includes nodes without enhanced card;

- MAC buffer must be shared among different channels in use, allowing for transmission on different channels, transfer between two channels at the same node (switching), as well as the traditional transfer of data.

MAC packet forwarding: our implementation, which executes routing operations at MAC, will be based on a next-hop address lookup in conjunction with a path strategy as, for example, the fixed length labels architecture as defined in [7]. Basically, the packet forwarding protocol builds on the IEEE 802.11 DCF MAC using RTS/CTS and uses some additional information in the control packets (RTS/CTS) to allow the forwarding node to determine the next hop node while contending for the channel. The nature of this additional information is matter of current research, as strongly related with the distribution of information among neighbors. Moreover, we intend to add channel information (as defined in previous point) for routing decisions.

Exportable parameters module: the architecture design foresees the access to MAC parameters for a full integration of mechanisms traditionally working at different layers. This is enabled by a cross-layering architecture as the one proposed for the MobileMAN project [10]. In this architecture the shared memory component acts as exchange area of networking information (parameters, status, etc...) for all the layers. As drown in Figure1, this allows the MAC layer to distribute "physical" information up to the higher levels, as well as to profit from some higher layers elaborations too complex to be performed at MAC. A typical example is the interaction between 
MAC, routing and transport information for congestion and network utilization purposes. If the transport is aware of the link status, it can distinguish between congestion due to physical failure and congestion due to the amount of traffic, and acts consequently. As well as the routing can decide different routing paths or strategies, and the MAC can modify the distribution of some information as consequence.

\section{Some Trials Scenarios}

We will implement the above described mechanisms and perform several levels of validation tests. Firstly, during the development phase, we will continuously test any new module inside the card in a stand-alone fashion and in communication with the other modules. Secondly, we will perform some 802.11 performance tests, similarly to [5]. Finally, we will put together some multi-hop wireless networks and test the workability of this solution. Some of the scenarios we plan to use are:

Ad hoc heterogeneous scenario - some of the ad hoc network nodes have not the enhanced MAC card: a) $\mathrm{N}$ is the only node with the enhanced card, it will select the standard backoff and use only one channel; b) $\mathrm{N}$ is not the only one with the enhanced card, $\mathrm{N}$ will use multiple channels: it will receive a multiplexed signal; agree with the peer(s) about the channel; select the enhanced backoff on the channels where this is possible. If the transmission goes through the LMFB for further forwarding, the MAC has to access the additional information about routing channels.

Ad hoc homogeneous scenario - all nodes in the ad hoc network use the enhanced MAC card: node $\mathrm{N}$ will use multiple channels and enhanced backoff: it will receive a multiplexed signal; select the channel with better backoff for transmitting.

Multi-hop WLAN heterogeneous scenario - some of the network nodes have not the enhanced MAC card: a) $\mathrm{N}$ is the only node with the enhanced card, it will select the standard backoff and, in the best case, use only channel 10 in ad hoc mode and 11 in WLAN mode; b) $\mathrm{N}$ is not the only one with the enhanced card, $\mathrm{N}$ will use multiple channels, (with a fixed one for the WLAN): it will receive a multiplexed signal; agree with the peer(s) about the channel; select the enhanced backoff on the channels where this is possible. If the transmission goes through the LMFB for further forwarding, the MAC has to access the additional information about routing channels.

Multi-hop WLAN homogeneous scenario - all nodes use the enhanced MAC card: node $\mathrm{N}$ will use multiple channels, (with a fixed one for the Access Point (AP)), and enhanced backoff: it will receive a multiplexed signal; select the channel with better backoff for transmitting.

\section{Conclusions}

In this paper, we presented the architecture for an enhanced IEEE 802.11 MAC card for improved performance in multi-hop wireless network, which is still fully compatible with current implementations. The main novel elements of this card are: 
- the access and management of operating channels, which allows for a more timely and efficient use of available capacity in several types of scenarios;

- a dynamically tuned backoff algorithm that allows to obtaining performance close to the optimum;

- a modular and exportable set of parameters for interaction with higher layers;

- a packet forwarding strategy integrated at MAC layer.

- This work has several open issues - among them:

- discovery mechanism for other nodes strategies (channels, backoff, routing, etc...);

- priority mechanisms;

- best additional information for performing MAC packet forwarding;

Future work includes the completion of the implementation (see [14] for more details), as well as a validation and a trial phases that will involve the higher layers for feedbacks.

\section{References}

1. IEEE Computer Society. 802.11: Wireless LAN Medium Access Control (MAC) and Physical Layer (PHY) Specifications, June 1997.

2. B. Crow, I. Widjaja, J. G. Kim and P. T. Sakai. IEEE 802.11: Wireless Local Area Networks. IEEE Communications Magazine, Sept 1999.

3. Xu Shugong and T. Saadawi: Does the IEEE 802.11 MAC Protocol Work Well in Multihop Wireless Ad Hoc Networks?, IEEE Communications Magazine, Jun 2001.

4. R. Bruno, M. Conti, and E. Gregori: Optimization of Efficiency and Energy Consumption in p-Persistent CSMA-Based Wireless LANs, IEEE Transaction on Mobile Computing, Jan 2002.

5. G. Anastasi, E. Borgia, M. Conti, and E. Gregori: IEEE 802.11 Ad Hoc Networks: Performance Measurements, Proceedings of MWN 2003.

6. R. Battiti, M. Conti, E. Gregori: Price-based Congestion-Control in Wi-Fi Hot Spots, Proceedings of Wiopt 2003.

7. A.Acharya, A. Minsra, S. Bansal: A label-switching packet forwarding architecture for multi-hop wireless LANs, Proceedings of Wowmom 2002.

8. S. Toumpis and A. J. Goldsmith: Performance, Optimization, and Cross-Layer Design of Media Access Protocols for Wireless Ad Hoc Networks, Proceedings of ICC 2003.

9. P. Stanforth and V. Hasty: Meshing Together: Advantages and Challenges of Deploying Ad Hoc Wireless, Networks http://grouper.ieee.org/groups/802/meeting/meeting_files/Ad\%20Hoc\%20And\%208022.pdf

10. M. Conti, S. Giordano, G. Maselli, G. Turi: MobileMAN Mobile Metropolitan ad hoc Network, pap, to appear in Proceedings of PWC 2003.

11. G. Bianchi, L. Fratta and M. Olivieri: Performance evaluation and enhancement of the CSMA/CA MAC Protocol for 802.11 Wireless LANs, Proceedings of PIMRC 1996.

12. F. Cali', M. Conti and E. Gregori: Dynamic IEEE 802.11: design, modeling and performance evaluation, IEEE Journal on Selected Areas in Communications, Sept. 2000.

13. R. Bernasconi, I. Defilippis, A. Puiatti: Issues and implementation plan of an enhanced MAC, draft version available at http://b.die.supsi.ch/projects/MobileMAN 